\title{
The Effects of Lichen Sclerosus and Its Treatments on Sexuality: Case Report
}

\author{
Janete Vettorazzi 1,2,3,4*, Edimárlei Gonsales Valério², Amanda Vilaverde Perez ${ }^{3}$, \\ Mariana Sbaraini ${ }^{3}$, Luciana Segat ${ }^{1}$, Daniela Vanessa Vetori ${ }^{1,4}$, Fernanda Santos Grossi ${ }^{1}$, \\ Valentino Antonio Magno ${ }^{1,2,3,4}$
}

\footnotetext{
${ }^{1}$ Postgraduation Program in Health Sciences: Gynecology and Obstetrics, Universidade Federal do Rio Grande do Sul (UFRGS). Rua Ramiro Barcelos, Santa Cecilia, Porto Alegre, RS, Brazil

${ }^{2}$ Department of Gynecology and Obstetrics, Faculty of Medicine (FAMED), UFRGS, Rua Ramiro Barcelos, Santa Cecilia, Porto Alegre, RS, Brazil

${ }^{3}$ FAMED, UFRGS, Rua Ramiro Barcelos, Santa Cecilia, Porto Alegre, RS, Brazil

${ }^{4}$ Service of Obstetrics and Gynecology, Hospital de Clínicas de Porto Alegre (HCPA), Rua Ramiro Barcelos, Santa Cecília, Porto Alegre, RS, Brazil

Email: ‘jvettorazzi@hcpa.edu.br, edimarleigv@terra.com.br, amandavperez@gmail.com, marisbaraini@gmail.com, dvvettori@gmail.com, lusegat@gmail.com, fernanda.grossi@yahoo.com.br,vmagno@hcpa.edu.br
}

How to cite this paper: Vettorazzi, J., Valério, E.G., Perez, A.V., Sbaraini, M., Segat, L., Vetori, D.V., Grossi, F.S. and Magno, V.A. (2019) The Effects of Lichen Sclerosus and Its Treatments on Sexuality: Case Report. Open Journal of Obstetrics and Gynecology, 9, 755-763.

https://doi.org/10.4236/ojog.2019.96075

Received: April 22, 2019

Accepted: May 28, 2019

Published: May 31, 2019

Copyright $\odot 2019$ by author(s) and Scientific Research Publishing Inc. This work is licensed under the Creative Commons Attribution International License (CC BY 4.0).

http://creativecommons.org/licenses/by/4.0/

\begin{abstract}
Lichen sclerosus is a chronic and autoimmune dermatitis that develops in the anogenital region. It is related to genital alterations with repercussions in sexuality. We present a case of a 60-year-old woman who had several chronic diseases and had been diagnosed with vulvar lichen sclerosus for 20 years, with no response to multiple treatments (topical corticosteroids, immunomodulators). She was submitted to vulvectomy; however, it leads to worsening of her condition, presenting obliterated and fibrosed clitoris, atrophic vaginal lips, and anal and vulvar fissures. She remained with dyspareunia, decreased sexual desire and difficulty in orgasm. She underwent sexual therapy, adjustment of antidepressant medication and made intercalated use of topical corticosteroids, topical testosterone and vaginal moisturizers, with partial improvement. From this case we can conclude that, although vulvectomy can be related to some degree of improvement in patients with lichen sclerosus, serious complications can occur.
\end{abstract}

\section{Keywords}

Lichen Sclerosus, Dyspareunia, Corticosteroids, Vulvectomy

\section{Introduction}

Lichen sclerosus or atrophic is a progressive and chronic dermatological condi- 
tion characterized by marked inflammation, epithelial thinning and specific dermal changes. It presents with symptoms of pain and itching. In $85 \%$ of the cases, it occurs in the anogenital region, but the disease can develop on any cutaneous surface [1]. The disease has two incidence peaks: pre-puberty, and perior postmenopause. It has an estimated prevalence of 1:30 in elderly women and an increased incidence in recent years [2]. The etiology of lichen sclerosus is unknown, and may be related to an immunological disorder with infectious and hormonal factors [3].

Lichen can lead to dysfunction and deconfiguration of the anogenital anatomy. The main therapy involves topical corticosteroids. In refractory cases, oral retinoids, phototherapy with UVA-1, topic estriol and topic testosterone can be used [4]. The risk of developing squamous cell vulvar carcinoma is around 5\% [5].

We report the case of a 60-year-old patient with atrophic vulvar lichen for 20 years, who underwent multiple drugs and surgical treatments, with partial response and repercussion in sexuality. The case occurred in the Hospital de Clínicas de Porto Alegre, in the state of Rio Grande do Sul, Brazil-a public-private institution linked to the Universidade Federal do Rio Grande do Sul.

\section{Case Report}

60 -year-old woman. She sought care at the sexology outpatient clinic at age 49 for complaints of dyspareunia and difficulty during intercourse. She had been diagnosed with lichen sclerosus at the age of 40 , after multiple biopsies. Patient had multiple comorbidities: hypertension, type 2 diabetes, depression, fibromyalgia, diverticular disease and nephrolithiasis. Chronic use of Metformin, Losartan, Furosemide, Metoprolol, Omeprazole, Simvastatin, Sertraline, Pregabalin, Clonazepam and Risperidone. She underwent total hysterectomy at age 40 due to menometrorrhagia and myomatosis.

At 46 years of age, the first simple vulvectomy was performed to improve lichen, without a satisfactory response. She used hormone replacement therapy with tibolone for 2 years, but maintained the hot flushes. At the age of 49 , she began follow up in the sexology clinic for dyspareunia and dissatisfaction with the aesthetic aspect of the vulva, assigning a note of 3 to sexuality on a scale of 1 to 10 . She presented with lichen in vulva and perianal region, and the clitoris was semi-fused. No improvement was obtained with use of topical testosterone in the genital region, but she had partial response to the use of tacrolimus and pimecrolimus. The patient continued treatment with clobetasol 3 times a week and started monthly $88 \mathrm{mg}$ of intramuscular testosterone. She reported increased libido, improved sexual satisfaction, decreased dyspareunia and increased clitoral volume with the intramuscular medication.

At age 50, she was evaluated by a surgical team that indicated a second vulvectomy. Anatomopathological exam performed in the procedure showed lichenoid interface dermatitis with mild subepidermal sclerosis and vascular neo- 
formation. There was partial improvement of the sexual complaints after the procedure. After 12 months, use of intramuscular testosterone was discontinued. The patient returned with symptoms of dyspareunia, absence of libido and perineal pain, therefore $100 \mathrm{mg}$ of intramuscular testosterone and $1 \mathrm{mg}$ of oral estradiol were reinstated, along with sporadic use of clobetasol. There was partial improvement of the libido, but persistence of dyspareunia and ulcerated lesions in the vulvar region. After presenting a significant increase of weight and body hair, the medication was suspended. An attempt to use topical tretinoin $0.025 \%$ was made, without patient tolerance. At age 52, she started using $2 \%$ testosterone cream in the clitorial region, showing an improvement in libido but permanence of vulvar pruritus and fissures.

After multiple treatments, the patient had her medications adjusted the sexology and psychiatry teams. She maintained use of topical clobetasol 3 times a week, oral sulpiride $50 \mathrm{mg}$ daily, intravaginal estriol 1 time a week, topic estriol in the vulva daily, and intravaginal moisturizing gel 2 times a week. In addition, she initiated sexual therapy with personal self-evaluation techniques, seeking pleasurable body parts outside the genital area and improving the intimacy of the couple. She had an increase in the libido, presence of orgasms and reduction of the vulvar lesions. At 55 years of age, she presented hot flushes, and daily 2.5 $\mathrm{mg}$ of tibolone and $2 \%$ testosterone vaginal cream were reinstated. She also received intramuscular testosterone every 40 days.

She maintained follow-up with the surgery, psychiatry and sexology clinics. At 56 years old, after complaints of vulvar aesthetics (vulvar atrophy, absence of small lips, flattening of the clitoris and fissures in the posterior fossa), the surgery team indicated lipofilling of the region. Suprapubic fat was removed, the material was centrifuged, and lipoenxertia on the vulva was made. On the ninth postoperative day, she developed cellulitis of difficult control. Despite antibiotic treatment with cefepime, she showed respiratory insufficiency and was admitted to the ICU. The antibiotic was changed to piperacillin and tazobactam, with improvement of clinical condition and discharge of ICU in 48 hours. In the postoperative period, there was an improvement in genital trophism, with a decrease in the lichen area. The patient maintained use of topical estrogen and testosterone in the vulvar region.

At age 57, she resumes follow-up at the sexology outpatient clinic, showing improvement in libido, but remaining with atrophic clitoris, fissures, and post-coital pain. Adjustments in the medications were made, maintaining tibolone $2.5 \mathrm{mg}$ a day, topical vaginal estriol 3 times a week, daily $2 \%$ testosterone cream applied in the clitoris, daily $2 \%$ testosterone gel in the abdomen and clobetasol when worsening of lichen. Currently, at the age of 60 , she reports having sexual intercourse about 2 to 3 times a month and orgasm in half of the times, with improvement in dyspareunia. In physical examination, there were no fissures in the perineum, and clitoris remained atrophic with whitish lesions characteristic of lichen (Figure 1). Compared to previous evaluations, the patient is 


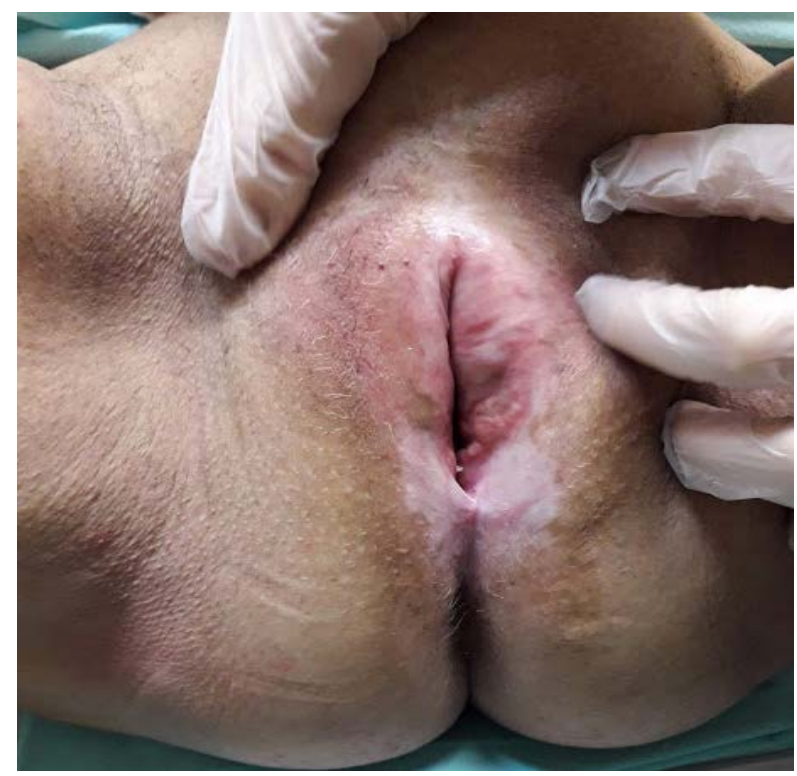

Figure 1. 60-year-old woman, 20 years of evolution with lichen sclerosus. Atrophic clitoris with whitish lesions characteristic of lichen.

currently in the best state of her illness and is satisfied with the treatment. The grade for sexuality on a scale of 0 to 10 is currently 7 . She continues her follow-up at the sexology outpatient clinic with consultations every six months. The patient authorized the disclosure of her case and signed a consent form. All authors are researchers from the medical institution and signed a term of data use, stating that the data was collected exclusively for scientific dissemination and will be presented without identification of the patient.

\section{Discussion}

Lichen sclerosus (LS) is a chronic inflammatory skin disease involving relapses and remissions, with a tendency to affect external genitalia. A prevalence of $1.7 \%$ was estimated in a recent study [2]. In $11 \%$ of the patients, it can affect extra-genital areas [6]. The disease can result in significant disfigurement of the vulva and, consequently, cause sexual dysfunction.

Etiology of the disease is still uncertain, but it is believed to involve genetic, hormonal, autoimmune and infectious mechanisms, thus being considered multifactorial [3] [4] [5] [7] [8] [9]. The histopathology of the disease is characteristic, making biopsy the best diagnostic tool. The skin displays atrophy of the epidermis, hydropic degeneration of the basal layer, edema, hyperkeratosis with follicular clogging, homogenization of the collagen in the upper dermis and inflammatory infiltrate in the form of a band formed by lymphoid cells mixed with plasmocytes and histiocytes [10] [11]. Localized or diffuse squamous hyperplasia is seen in $2 / 3$ of the patients, resulting from chronic friction or itching [12]. One study credits the white coloration of lesions to decreased melanin production, transfer of melanosomes to keratinocytes and loss of melanocytes [13].

The main symptoms reported in lichen are vulvar pruritus and irritation, but 
there may be dyspareunia, dysuria, burning sensation and intestinal problems. The extragenital lesions are asymptomatic and mainly affect inner thigh area, infra-mammary region, neck, shoulders and wrists. Patients with advanced disease may present narrow vaginal introitus, making intercourse even more difficult. Sexual discomfort is due to the physical effects of LS, since the disease causes fissures, erosions, scars, labial fusion and introital stenosis. Genital atrophy can lead to loss of the small labia, destruction of the clitoris and obstruction of the urinary stream. In addition, many women with LS are dissatisfied with the appearance of the vulva, which amplifies discomfort during sexual intercourse [14]. In the presented case, in addition to all the typical symptomatology, the patient had important complaints regarding vulvar aesthetics, which was another obstacle in her sexuality.

In LeFevre's study [15], LS patients with dyspareunia and vulvar pain/burning had complete relief of symptoms after treatment in $45 \%$ to $92 \%$ of the cases. Although topical treatments are effective for symptomatic relief and histopathological improvement, sexual dysfunction persist in many women [14]. In our case, although remission of symptoms such as pain and pruritus had occurred, sexual dysfunction was a constant, showing little improvement. Cheng and colleagues [16] demonstrated that $63 \%$ of the patients with LS had sexual dysfunction. Quality of life is also extremely affected in this disease. Numerous studies using questionnaires [17] [18] [19] showed that LS affected all the main domains of quality of life, except work/study. A Dutch paper [20] compared the sexual function of women with LS to those of a control group. Patients with lichen presented a mean Female Sexual Function Index (FSFI) score of 18.79 and had lower points in all categories of the questionnaire, while the control group had a mean FSFI of 27.43. Female Sexual Function and Dysfunction (FSDS) scores indicated sexual dysfunction only in patients with LS. In the reported case, our patient had a significant improvement in sexuality and quality of life after vulva lipofilling. Although no specific questionnaire was applied, the grade for sexuality improved from 3 to 7 (on a scale of 0 to 10 ).

Sexual difficulties in LS patients can be attributed to three main causes of dyspareunia. First, the skin is sensitive and delicate, and lesions can easily arise and cause dyspareunia. Second, fear of pain diminishes sexual arousal and vaginal lubrication, and leads to muscle contraction, making intercourse more difficult. Third, the anatomical changes that occur in LS, such as large lips fusion and introitus stenosis, makes sexual intercourse painful and orgasm more difficult. All these factors were present in the reported patient, which hindered her sexual relations.

Treatment should be implemented in asymptomatic as well as symptomatic patients, aiming relief of symptoms and discomfort; preventing anatomical changes and preventing (theoretically) malignant transformation. Ultrapotent corticosteroids are the treatment of choice for adults. Clobetasol propionate $0.05 \%$ is the most frequently used and, according to some studies [21], can reduce the symptoms in $77 \%$ of the patients. There is no standard application re- 
gimen, but it is common to start with one application a day for 3 months. After an initial therapy of 6 months, some may use the corticosteroid only when needed, while others will have to continue therapy twice a week. Long-term treatment with corticosteroids can cause atrophy and telangiectasia. The use of intralesional corticosteroids is an alternative in cases of thick plaques resistant to topical corticosteroids. Monthly injections of triamcinolone acetonide 5 to 20 $\mathrm{mg}$ per $\mathrm{ml}$ are applied in vulvar LS lesions. Triamcinolone have the same efficacy as clobetasol, but fewer systemic and local adverse effects. Our patient had partial response to corticosteroids, remaining in chronic use of the medication, especially during relapses of the disease.

In some studies [22] [23], topic testosterone showed a poor efficacy, no longer being considered as one of the first treatment options. However, in cases of multiresistant patients, it may present as an alternative. In the patient of the reported case, there was improvement with use of topical testosterone without the occurrence of important adverse effects, since the applied dose was low. Systemic retinoids have been used with some success in complicated lichen without response to corticosteroids, but side effects limit their use. Topical retinoids have already been used as an alternative, but skin irritation after application is an important limiting factor [24]. Our patient did not tolerate the use of this medication.

Treatment with $0.1 \%$ Tacrolimus ointment is an alternative. It is an immunosuppressive agent used to prevent rejection after transplantation. It acts by inhibiting calcineurin, which inhibits the transcription of interleukin-2, interferon and other proinflammatory cytokines; thus suppressing the activation and differentiation of $\mathrm{T}$ cells and other inflammatory cells. Because of its lipophilic nature, tacrolimus penetrates easily into damaged skin. The medication also suppresses mast cells and basophils, which decreases pruritus. A positive side of the medication is that it does not affect keratinocyte proliferation or collagen synthesis, therefore not causing atrophy like topical corticosteroids. A prospective 2002 study showed improvement of pruritus and pain, in addition to complete remission in $70 \%$ of the patients using $0.1 \%$ Tacrolimus ointment twice a day [25]. Currently, tacrolimus is the second treatment option only in case of failure or contraindication to clobetasol, since corticosteroids are more effective in reducing inflammation. The presented patient showed no response to $\mathrm{Ta}$ crolimus.

Surgery should not be performed in cases of uncomplicated LS. It should be done in patients with associated malignancy, for correction of scars secondary to disease or to widen the vaginal introitus due to difficulties in urination or intercourse. However, prior to any surgical intervention, LS should be clinically controlled. Vulvoperineoplasty for introital stenosis involves transposition of vaginal mucosa into the introitus, considering that the vaginal epithelium is not affected by the disease. Vulvar lipofilling is a fat grafting technique that has been implemented in recent years. In our patient, it was made as an attempt to im- 
prove visual aesthetics and, consequently, sexuality. Some techniques still under study are cryotherapy and carbon dioxide laser [26] [27].

Patients should be seen by a doctor at least twice a year due to the risk of squamous cell carcinoma-LS is associated with a $4 \%$ to $6 \%$ risk of malignancy [14]. Squamous cell carcinoma lesions are typically found in the clitoris and labia minora. The presented patient has a multidisciplinary follow-up, visiting every 6 months with the teams of gynecological oncology, dermatology and sexology.

For sexual dysfunction, although there are no randomized clinical studies, the usual recommendation is to perform regular consultations with sexologists and to use lubricants for reducing friction. Although estrogen-based creams are not effective in treating LS, they can be used to improve skin tropism and to decrease discomfort during intercourse. Drug treatment may improve but not eradicate sexual symptoms.

Patients may develop sensitivity changes (dysesthesia) and these may remain even after improvement of skin lesions. In these cases, patients show no response to corticosteroids because pain is neuropathic, therefore an alternative is the use of 5\% lidocaine ointment. In non-responsive cases, amitriptyline or gabapentin (that have a pain inhibitory effect in the central nervous system) can be used [28].

\section{Conclusion}

There is little medical literature about the consequences of lichen sclerosus on women's sexual lives. Existing treatments are known to relieve symptoms such as pain and itching, but do not completely resolve sexual dysfunction. Although top-line treatment is topical corticosteroids, second line alternatives are also effective-such as topical tacrolimus, an immunosuppressant capable of reducing local inflammation. Surgery should be performed only in cases refractory to all drug therapies, since it does not always have satisfactory results and may worsen the disease due to the Koebner phenomenon. In our reported case, we show a patient who presented symptomatic improvement of LS after numerous treatments, but remained with sexual dysfunction. At this point, lipofilling had a satisfactory response by improving vaginal esthetics, an important complaint of the patient. Lichen planus is a chronic multisystemic disease of difficult management; and the presence of physiotherapists, psychologists, dermatologists, gynecologists and specialists in sexual health is fundamental. Performing radical surgeries may increase the risk of complications in short and long terms and do not bring benefit to the patient. We emphasize the importance of follow up of this patient with a multidisciplinary team.

\section{Acknowledgements}

We would like to express our deepest gratitude to the participants for their time and patience throughout this study. 


\section{Financial Disclosure}

The authors have no financial relationships relevant to this article to disclose.

\section{Conflicts of Interest}

The authors have no conflicts of interest to disclose.

\section{References}

[1] Powell, J. and Wojnarowska, F. (2001) Childhood Vulvar Lichen Sclerosus: An Increasingly Common Problem. Journal of the American Academy of Dermatology, 44, 803-806. https://doi.org/10.1067/mjd.2001.113474

[2] Goldstein, A.T., Marinoff, S.C., Christopher, K. and Srodon, M. (2005) Prevalence of Vulvar Lichen Sclerosus in a General Gynecology Practice. Journal of Reproductive Medicine, 50, 477-480.

[3] Marren, P., Yell, J., Charnock, F.M., et al. (1995) The Association between Lichen Sclerosus and Antigens of the HLA System. British Journal of Dermatology, 132, 197-203. https://doi.org/10.1111/j.1365-2133.1995.tb05013.x

[4] Sideri, M., Origoni, M., Spinaci, L. and Ferrari, A. (1994) Topical Testosterone in the Treatment of Vulvar Lichen Sclerosus. International Journal of Gynecology \& Obstetrics, 46, 53-56. https://doi.org/10.1016/0020-7292(94)90309-3

[5] Derrick, E.K., Ridley, C.M., Kobza-Black, A., Mckee, P.H. and Neill, S.M. (2000) A Clinical Study of 23 Cases of Female Anogenital Carcinoma. British Journal of Dermatology, 143, 1217-1223. https://doi.org/10.1046/j.1365-2133.2000.03891.x

[6] Funaro, D. (2004) Lichen Sclerosus: A Review and Practical Approach. Dermatologic Therapy, 17, 28-37. https://doi.org/10.1111/j.1396-0296.2004.04004.x

[7] Haefner, H.K., Aldrich, N.Z., Dalton, V.K., Gagne, H.M., Marcus, S.B., Patel, D.A. and Berger, M.B. (2014) The Impact of Lichen Sclerosus on Sexual Dysfunction. Journal of Women's Health, 23, 765-770. https://doi.org/10.1089/jwh.2014.4805

[8] Purcell, K.G., Spencer, L.V., Simpson, P.M., Helman, S.W., Oldfather, J.W. and Fowler Jr., J.F. (1990) HLA antigens in lichen sclerosus et atrophicus. Archives of Dermatology, 126, 1043-1045.

[9] Clay, F.E., Cork, M.J., Tarlow, J.K., et al. (1994) Interleukin 1 Receptor Antagonist Gene Polymorphism Association with Lichen Sclerosus. Human Genetics, 94, 407-410. https://doi.org/10.1007/BF00201602

[10] Goolamali, S.K., Barnes, E.W., Irvine, W.J. and Shuster S. (1974) Organ Specific Antibodies in Patients with Lichen Sclerosus. The BMJ, 4, 78-79. https://doi.org/10.1136/bmj.4.5936.78

[11] Meyrick Thomas, R.H., Ridley, C.M., McGibbon, D.H. and Black, M.M. (1988) Lichen Sclerosus and Autoimmunity. A Study of 350 Women. British Journal of Dermatology, 118, 41-46. https://doi.org/10.1111/j.1365-2133.1988.tb01748.x

[12] Oyama, N., Chan, I., Neill, S.M., et al. (2003) Autoantibodies to Extracellular Matrix Protein 1 in Lichen Sclerosus. The Lancet, 362, 118-123. https://doi.org/10.1016/S0140-6736(03)13863-9

[13] Carlson, J.A., Grabowski, R., Mu, X.C., Del Rosario, A., Malfetano, J. and Slominski, A. (2002) Possible Mechanisms of Hypopigmentation in Lichen Sclerosus. The American Journal of Dermatopathology, 24, 97-107. https://doi.org/10.1097/00000372-200204000-00001 
[14] Dalziel, K.L. (1995) Effect of Lichen Sclerosus on Sexual Function and Parturition. Journal of Reproductive Medicine, 40, 351-354.

[15] Le Fevre, C., Hoffstetter, S., Meyer, S. and Gavard, J. (2011) Management of Lichen Sclerosus with Triamcinolone Ointment: Effectiveness in Reduction of Patient Symptom Scores. Journal of Lower Genital Tract Disease, 15, 205-259. https://doi.org/10.1097/LGT.0b013e31820a3b6f

[16] Cheng, H., Oakley, A., Conaglen, J. and Conaglen, H. (2016) Quality of Life and Sexual Distress in Women with Erosive Vulvovaginal Lichen Planus. Journal of Lower Genital Tract Disease, 21, 145-149. https://doi.org/10.1097/LGT.0000000000000282

[17] Neill, S.M., Tatnall, F.M. and Cox, N.H. (2002) Guidelines for the Management of Lichen Sclerosus. British Journal of Dermatology, 147, 640-649. https://doi.org/10.1046/j.1365-2133.2002.05012.x

[18] Tasker, G.L. and Wojnarowska, F. (2003) Lichen Sclerosus. Clinical and Experimental Dermatology, 28, 128-133. https://doi.org/10.1046/j.1365-2230.2003.01211.x

[19] Rajagopalan, R., Anderson, R., Edwards, L. and Sherertz, E. (1999) Quality of Life Evaluation in Chronic Lichen Sclerosus for Improved Medical Care. Therapeutic Innovation \& Regulatory Science, 33, 577-584. https://doi.org/10.1177/009286159903300228

[20] Van de Nieuwenhof, H.P., Meeuwis, K.A., Nieboer, T.E., Vergeer, M.C., Massuger, L.F. and De Hullu, J.A. (2010) The Effect of Vulvar Lichen Sclerosus on Quality of Life and Sexual Functioning. Journal of Psychosomatic Obstetrics and Gynecology, 31, 279-284.https://doi.org/10.3109/0167482X.2010.507890

[21] Lorenz, B., Kaufman, R.H. and Kutzner, S.K. (1998) Lichen Sclerosus. Therapy with Clobetasol Propionate. Journal of Reproductive Medicine, 43, 790-794.

[22] Cattaneo, A., Carli, P., De Marco, A., Sonni, G., De Magnis, A. and Taddei, G.L. (1996) Testosterone Maintenance Therapy. Effects on Vulvar Lichen Sclerosus Treated with Clobetasol Propionate. Journal of Reproductive Medicine, 41, 99-102.

[23] Bornstein, J., Heifetz, S., Kellner, Y., Stolar, Z. and Abramovici, H. (1998) Clobetasol Propionate $0.05 \%$ versus Testosterone Propionate $2 \%$ Topical Application for Severe Vulvar Lichen Sclerosus. American Journal of Obstetrics \& Gynecology, 178, 80-84. https://doi.org/10.1016/S0002-9378(98)70631-3

[24] Bracco, G.L., Carli, P., Sonni, L., et al. (1993) Clinical and Histological Effects of Topical Treatments of Vulval Lichen Sclerosus: A Critical Evaluation. Journal of Reproductive Medicine, 38, 37-40.

[25] Böhm, M., Frieling, U., Luger, T.A. and Bonsmann, G. (2003) Successful Treatment of Anogenital Lichen Sclerosus with Topical Tacrolimus. Archives of Dermatology, 139, 922-924. https://doi.org/10.1001/archderm.139.7.922

[26] August, P.J. and Milward, T.M. (1980) Cryosurgery in the Treatment of lichen sclerosus et atrophicus of the Vulva. British Journal of Dermatology, 103, 667-670. https://doi.org/10.1111/j.1365-2133.1980.tb01690.x

[27] Kartamaa, M. and Reitamo, S. (1997) Treatment of Lichen Sclerosus with Carbon dioxide Laser Vaporization. British Journal of Dermatology, 136, 356-359. https://doi.org/10.1046/j.1365-2133.1997.d01-1199.x

[28] Pinelli, S., D’Erme, A.M. and Lotti, T. (2013) Management of Sexual Dysfunction Due to Vulvar Lichen Sclerosus in Postmenopausal Women. Dermatologic Therapy, 26, 79-82. https://doi.org/10.1111/j.1529-8019.2012.01536.x 